\title{
COMPRESSIBILIDADE DE UM SOLO SOB SISTEMAS DE PASTEJ O ROTACIONADO INTENSIVO IRRIGADO E NÃO IRRIGADO(1)
}

\author{
C. L. R. LIMA(2), A. P. SILVA(3), S. IMHOFF(4) \& T. P. LEÃO(5)
}

\begin{abstract}
RESUMO
O incremento da produtividade das pastagens tem sido associado ao comportamento à compressão dos solos. Os objetivos deste trabalho foram: quantificar as curvas de compressão e a pressão de preconsolidação em sistemas de pastejo intensivo rotacionado irrigado e não irrigado. Foram coletadas 96 amostras indeformadas de solo em quatro ciclos sucessi vos de pastejo instalado com capim Tanzânia (Panicum maximumJ acq.) em um Argissolo Vermelho. Após saturadas com água e equilibradas no potencial $(\psi)$ : -10 kPa, as amostras foram pesadas e submetidas ao ensaio de compressão uniaxial com a aplicação sucessiva e contínua de pressões de 25, 50, 100, 200, 400, 600, 800, 1.000, 1.300 e $1.600 \mathrm{kPa}$. Os resultados comprovaram a hipótese de que houve diferença na compressibilidade do solo sob os sistemas de pastejo rotacionado intensivo irrigado e não irrigado. A maior compactação inicial verificada no sistema de pastejo rotacionado intensivo irrigado favoreceu o deslocamento das curvas de compressão uniaxial para valores superiores de densidade do solo. A pressão de preconsolidação foi significativamente superior no quarto ciclo de pastejo no sistema de pastejo rotacionado intensivo irrigado em relação ao sistema pastejo rotacionado intensivo não irrigado.
\end{abstract}

Termos de indexação: compactação do solo, curvas de compressão uniaxial, pressão de preconsolidação.

\footnotetext{
(1) Parte da Tese de Doutorado do primeiro autor. Recebido para publicação em dezembro de 2003 e aprovado em outubro de 2004.

(2) Pós-Doutoranda em Ciência do Solo, Universidade Federal de Santa Maria - UFSM. Faixa de Camobi, Km 9, CEP 97105-900 Santa Maria (RS). E-mail: clrlima@mail.ufsm.br

(3) Professor do Departamento de Solos e Nutrição de Plantas, Escola Superior de Agricultura "Luiz de Queiroz" - ESALQ/USP. Av. Pádua Dias 11, Caixa Postal 9, CEP 13418-900 Piracicaba (SP). E-mail: apisilva@esalq.usp.br

(4) Professora da Universidad Nacional Del Litoral, Avenida P. Kreder 2805, CEP 3080, Esperanza, Argentina. E-mail: simhoff@fca.unl.edu.ar

(5) Doutorando em Solos e Nutrição de Plantas, ESALQ/USP. E-mail: tpleao@esalq.usp.br
} 


\title{
SUMMARY: SOIL COMPRESSIBILITY UNDER NON-IRRIGATED AND IRRIGATED SHORT DURATION GRAZING SYSTEMS
}

\begin{abstract}
Increment in pasture productivity has been associated with the understanding of the soil behavior under compression. The objective of this research was to quantify (a) the compression curves and (b) the preconsolidation pressure of the soils under non-irrigated and irrigated short duration grazing systems. Ninety-six undisturbed soil samples were taken from thefour successive pasture cycles of Tanzania grass (Panicum maximum J acq.) in a Hapludalf. Thesamples weresaturated in water and equilibrated at thematrix potential $(\psi):-10 \mathrm{kPa}$ and then wereweighed and submitted to an uniaxial compression test, applying thefollowing pressures: $25,50,100,200,400,600,800,1,000,1,300$, and 1,600 kPa. Results support the hypothesis that thereis a difference between the soil compressibility under nonirrigated and irrigated short-duration grazing systems. The higher initial compaction verified in irrigated short-duration grazing system favored the displacement of theuniaxial compression curves for higher bulk density values. The preconsolidation pressure was significantly higher in thefourth pasturecycleunder irrigated than under thenon-irrigated short duration grazing system.
\end{abstract}

Index terms: soil compaction, uniaxial compression curves, preconsolidation pressure.

\section{NTRODUÇÃO}

O Brasil abrange uma área total de pastagens na ordem de 180 milhões de hectares, o que corresponde a mais de $20 \%$ do território brasileiro (Zimmer et al., 2002). Um dos grandes entraves na obtenção de el evadas produtividades tem sido a compactação do solo. A compressibilidade do solo, que indica a resistência do solo ao decréscimo de volume quando submetido a uma dada pressão (Horn \& Lebert, 1994), é determinada não só pelas características do solo, como a textura ea umidade, mas também pela intensidade, freqüência e tipo de sistema de pastejo adotado (Stephenson \& Veigel, 1987; Defossez \& Richard, 2002).

$\mathrm{Na}$ literatura, há indicações de que os animais podem exercer pressões no solo da ordem de 350 a 400 kPa (Proffitt et al., 1993; Betteridge et al., 1999). Nie et al. (2001) indicaram ainda que a pressão exercida pelos animais em movimento pode ser o dobro da exercida quando el es estão parados.

Diversas técnicas têm sido utilizadas no intuito de obter incremento da produção das pastagens, como as técni cas de adubação, a adoção de diferentes sistemas de pastejo e, ultimamente, a irrigação (Chanasyk \& N aeth, 1995). No entanto, a irrigação em pastagens pode facilitar a compactação do solo, uma vez que os mai ores efeitos do pisoteio de animais são evidenciados principalmente quando o solo encontra-se com umidade elevada (Tanner \& Mamaril, 1959; Betteridge et al., 1999).

A densidade, a resistência do solo à penetração e a porosidade são al guns dos indicadores utilizados na avaliação da compactação do solo em áreas sob pastagens (Ferrero, 1991; Proffitt et al., 1993; Silva et al., 2003). Esses indicadores caracterizam-se por aval iar o estado de compactação, mas não aval iam o processo de compressão do solo.

O comportamento compressivo do solo baseia-se na avaliação das curvas de compressão uniaxial. A pressão de preconsolidação do solo, propriedade obtida da curva de compressão, tem sido amplamente aceita como um indicativo de sua capacidade de suporte, uma vez que a aplicação de pressões maiores a este valor podem provocar a compactação adicional ao solo (Dias) únior \& Pierce, 1996; Kondo \& Dias J únior, 1999).

No Brasil, há carência de estudos que descrevam o comportamento compressivo de solos irrigados, cultivados com pastagens e submetidos à utilização intensiva. A hipótese testada neste estudo é de que há diferença na compressibilidade de solos sob os sistemas de pastejo rotacionado intensivo irrigado e não irrigado. O objetivo deste trabalho foi quantificar as curvas de compressão e a pressão de preconsolidação em sistemas de pastejo intensivo rotacionado irrigado e não irrigado.

\section{MATERIAL E MÉTODOS}

O estudo foi realizado em uma área experimental da ESALQ/USP Iocalizada na Fazenda Areão, Piracicaba, SP (22 42 ' 30 " S; 47 ० 38 ' 30 " W; $576 \mathrm{~m})$. O clima é caracterizado como Cwa (mesotérmico úmido subtropical de inverno seco) de acordo com a classificação deK öppen. A precipitação pluvial média anual é de $1.253 \mathrm{~mm}$, com as chuvas concentradas no período de novembro a fevereiro. A temperatura média anual éde $21^{\circ} \mathrm{C}$, com a média 
do mês mais frio inferior a $18{ }^{\circ} \mathrm{C}$ e a média do mês mais quente superior a $22^{\circ} \mathrm{C}$. A área encontra-se cultivada com capim Tanzânia (Panicum maximum J acq.), estabel ecido em um Argissolo Vermelho (Embrapa, 1999).

A área total doexperimento foi de 3,2 ha, dividida em oito blocos de 0,4 ha. Cada bloco dispunha de três piquetes de 0,133 ha, totalizando 24 piquetes. As áreas analisadas foram: uma irrigada com pivô central, e outra não irrigada, cada uma composta por quatro blocos e 12 piquetes.

O ciclo do pastejo, os períodos de descanso e de ocupação foram de 36, 33 e 3 dias, respectivamente. A lotação animal foi ajustada em todos os piquetes para deixar uma quantidade de resíduo remanescente de $2.500 \mathrm{~kg} \mathrm{MS} \mathrm{ha}^{-1}$.

O preparo do solo consistiu de uma gradagem aradora e duas gradagens niveladoras. A semeadura foi realizada em linhas no mês de março de 1999. Para o estabel ecimento das pastagens, foi efetuada a correção do solo por meio de calagem e adubação. O primeiro pastejo foi real izado no mês de agosto do ano de 2001.

Para o manejo da irrigação, foram instaladas cinco baterias de tensiômetros nas profundidades de 0,$10 ; 0,30 ; 0,50 ; 0,70$ e 0,90 m. A irrigação foi iniciada, quando os tensiômetros registravam um potencial de $(\Psi)$ : -30 a -40 kPa, e finalizada em um potencial de $(\Psi)$ : $-10 \mathrm{kPa}$.

As amostragens foram feitas em quatro ciclos sucessivos correspondentes aos meses de novembro, dezembro, janeiro e feverei ro dos anos de 2001 e2002, respectivamente. Em cada piquete, foi retirada, por ciclo, na camada superficial, uma amostra com estrutura indeformada, com anel volumétrico de 0,07 m de diâmetro e $0,025 \mathrm{~m}$ de altura, totalizando 96 amostras.

As amostras foram saturadas com água por $48 \mathrm{~h}$ e equilibradas no potencial $(\Psi)$ : -10 kPa em câmaras de Richards (Klute, 1986). Após o equilíbrio, cada amostra foi pesada e submetida ao ensaio de compressão uniaxial, que consistiu na aplicação sucessiva e contínua de pressões crescentes e preestabel ecidas de 25, 50, 100, 200, 400, 800, 1.000, 1.300 e $1.600 \mathrm{kPa}$, por cinco minutos, período suficiente para atingir 99 \% da deformação máxima (Silva et al., 2002). O desl ocamento vertical ocorrido em cada pressão aplicada foi registrado por meio de um sensor ligado a um sistema de aquisição de dados automatizado M CT 2000 (MIRAE Engineering, Inc., Buscan, Korea). O ensaio permitiu obter a curva de compressão do solo. As amostras foram secas em estufa a $105^{\circ} \mathrm{C}$, por $24 \mathrm{~h}$, e, a partir destes valores, foi determinada para cada amostra a densidade do solo $\left(D_{s}\right)$ (Blake\& Hartge, 1986). A partir das curvas de compressão uniaxial, foram obtidos os val ores de pressão de preconsolidação (Casagrande, 1936), utilizando o software Mathcad (Mathsoft I nc., 2000), conforme I mhoff et al. (2004).
Os valores médios da pressão de preconsol idação, para os sistemas de pastejo intensivo rotacionado irrigado $\left(P R I_{I}\right)$ e não irrigado $\left(P R I_{N I}\right)$, foram comparados, utilizando o teste t de student a $5 \%$ (SAS, 1991).

\section{RESULTADOS E DISCUSSÃO}

Os momentos estatísticos das variáveis analisadas sob os sistemas de pastejo rotacionado intensivo irrigado $\left(\mathrm{PRI}_{1}\right)$ e não irrigado $\left(\mathrm{PRI} \mathrm{NI}_{\mathrm{N}}\right)$ estão apresentados no quadro 1. A amplitude de variação da umidade com base gravimétrica esteve possivel mente relacionada com o intervalo de variação do teor argila em cada área de pastejo.

A pressão de preconsolidação, considerada um indicador da pressão máxima a que o solo foi submetido anteriormente, apresentou, nos dois sistemas, valores médios semelhantes aos obtidos por Kondo \& Dias J únior (1999) em solos sob pastagens. Por outro lado, foram superiores aos valores encontrados por Silva et al. (2002) em um solo sob as culturas de soja e de milho. Estas diferenças podem ser atribuídas ao fato de o gado aplicar el evadas pressões ao solo, podendo superar os valores de pressões exercidas por máquinas agrícolas convencionais (M apfumo et al., 1999), bem como à ausência de revolvimento da camada superficial do sol o cultivado com pastagens, operação que normalmente ocorre no cultivo de culturas anuais.

O intervalo de variação da pressão de preconsolidação nas duas áreas analisadas foi semelhante aos valores da pressão exercida por animais da raça bovina, indicados na literatura científica (Willatt \& Pullar, 1983; Betterigde et al., 1999).

O intervalo de variação da densidade do solo indicou ampla variabilidade espacial do estado de compactação, provavel mente ocasionada pelas condições diferenciadas de umidade nos dois sistemas $\left(P R I_{1}\right.$ e $\left.P R I_{N I}\right)$ (irrigação). O maior valor médio de densidadeobservado no sistema dePRI, reflete o efeito da umidade, ou seja, da implementação da técnica da irrigação sobrea compactação do sol o no momento do pisoteio do gado, corroborando os resultados apresentados por Ferrero (1991), Proffitt et al. (1993) e J orajuria et al. (1997).

Os valores médios da $\sigma_{\mathrm{p}}$ em cada ciclo, nos sistemas de $P R I_{1}$ e $P R I_{N I}$ estão apresentados na figura 1. Os valores da $\sigma_{\mathrm{p}}$ foram maiores no sistema de $P R I_{1}$, indicando que o solo foi submetido a um maior estresse nesse sistema. A umidade do sol ono momento do pisoteio, mais elevada nesse sistema (irrigação), pode ter contribuído para o aumento significativo da compactação inicial no sistema de

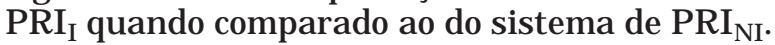


Quadro 1. Momentos estatísticos das variáveis analisadas sob os sistemas de pastejo rotacionado intensivo irrigado $\left(P R I_{1}\right)$ e não irrigado $\left(P R I_{N I}\right)$

\begin{tabular}{|c|c|c|c|c|c|c|}
\hline Variável(1) & $\mathbf{n}$ & Média & Desvio-padrão & Mínimo & Máximo & C.v. (\%) \\
\hline & \multicolumn{6}{|c|}{$P R I_{1}$} \\
\hline Argila, $\mathrm{g} \mathrm{kg}^{-1}$ & 12 & 425,83 & 32,87 & 370,00 & 480,00 & 7,72 \\
\hline Areia, $\mathrm{g} \mathrm{kg}^{-1}$ & 12 & 329,17 & 47,12 & 250,00 & 410,00 & 14,32 \\
\hline Silte, $\mathrm{g} \mathrm{kg}^{-1}$ & 12 & 235,00 & 34,39 & 190,00 & 290,00 & 14,64 \\
\hline $\mathrm{Ug}, \mathrm{kg} \mathrm{kg}^{-1}$ & 48 & 0,18 & 0,01 & 0,15 & 0,20 & 6,77 \\
\hline$\sigma_{p}, \mathrm{kPa}$ & 48 & 268,00 & 86,49 & 107,00 & 466,00 & 32,25 \\
\hline \multirow[t]{2}{*}{ Ds, $\mathrm{Mg} \mathrm{m}^{-3}$} & 48 & 1,68 & 0,08 & 1,53 & 1,83 & 4,62 \\
\hline & \multicolumn{6}{|c|}{$P R I_{N I}$} \\
\hline Argila, $\mathrm{g} \mathrm{kg}^{-1}$ & 12 & 517,50 & 42,50 & 440,00 & 580,00 & 8,21 \\
\hline Areia, $\mathrm{g} \mathrm{kg}^{-1}$ & 12 & 267,50 & 32,26 & 220,00 & 330,00 & 12,06 \\
\hline Silte, $\mathrm{g} \mathrm{kg}^{-1}$ & 12 & 216,67 & 20,97 & 180,00 & 250,00 & 9,91 \\
\hline $\mathrm{Ug}, \mathrm{kg} \mathrm{kg}^{-1}$ & 48 & 0,22 & 0,02 & 0,15 & 0,25 & 11,20 \\
\hline$\sigma_{\mathrm{p}}, \mathrm{kPa}$ & 48 & 246,00 & 82,12 & 104,00 & 438,00 & 33,44 \\
\hline $\mathrm{D}_{\mathrm{s}}, \mathrm{Mg} \mathrm{m}^{-3}$ & 48 & 1,61 & 0,08 & 1,45 & 1,75 & 4,83 \\
\hline
\end{tabular}

(1) $\mathrm{Ug}=$ umidade com base gravimétrica; $\sigma_{\mathrm{p}}=$ pressão de preconsolidação; $\mathrm{D}_{\mathrm{s}}=$ Densidade do solo.

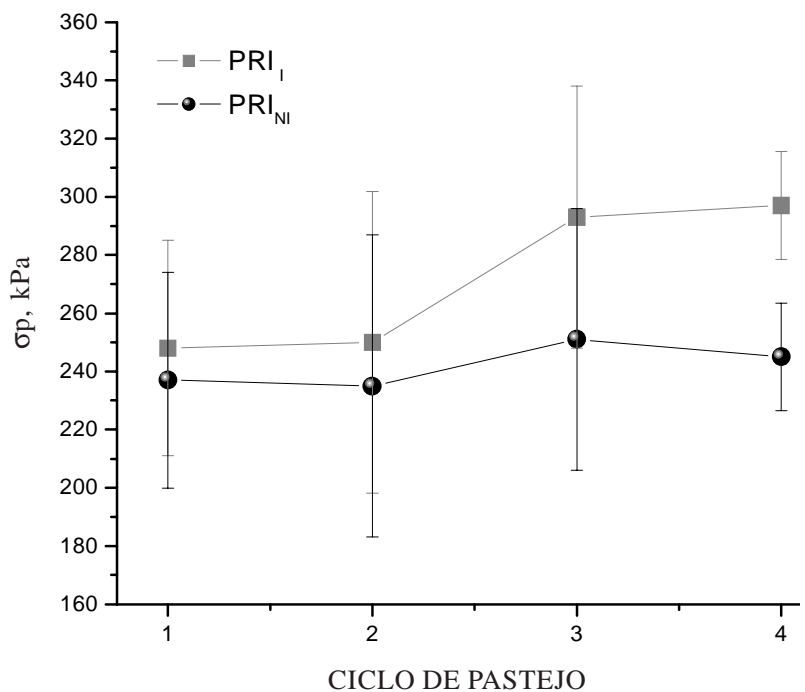

Figura 1. Valores médios da pressão de preconsolidação do solo $\left(\sigma_{\mathrm{p}}\right)$ em cada ciclo sob os sistemas de pastejo rotacionado intensivo irrigado $\left(P R I_{1}\right)$ e não irrigado $\left(P R I_{N I}\right)$. Barras verticais indicam a diferença mínima significativa a $5 \%$ entre os sistemas de PRI, e PRI ${ }_{\mathrm{NI}}$ :

Os animais aplicam elevadas pressões no solo porque o seu peso é distribuído numa área (o casco) muito pequena. Além disso, os animais não permanecem estáticos na área de pastejo. A pressão que os animais exercem sobre o solo também pode estar relacionada com a energia cinética que é transmitida ao solo, quando estes estão em movimento (Willatt \& Pullar, 1983). Um animal em movimento é capaz de realizar um desl ocamento ou uma deformação em outro corpo (solo). A força atribuída a este movimento é chamada de energia cinética, a qual está diretamente relacionada com a massa (peso do animal) e com a velocidade de movimentação. Abayeet al. (1997) indicaram quea força dinâmica exercida por um animal sobre o solo é aproximadamente o dobro da força estática, o que corrobora as observações de Nie et al. (2001).

A ausência de diferença significativa até o terceiro ciclo de pastejo pode estar relacionada com a variação dos dados. A di minuição da variação da $\sigma_{\mathrm{p}}$, no decorrer do tempo, avaliada por meio do coeficiente de variação, justificou a obtenção de diferenças significativas desta propriedadeentre os dois sistemas analisados no último ciclo de pastejo (Figura 2).

As diferenças da $\sigma_{p}$ podem estar associadas ao efeito do pisoteio dos ani mais eao tempo de avaliação do experimento. Considerando o fato de os animais não permanecerem estáticos na área, é comum observar em sistemas de pastejo pontos mais compactados (correspondentes às marcas dos cascos) distribuídos desuniformemente no solo.

Por outro lado, é de se esperar que, com os sucessivos ciclos de pastejo, os animais pisoteiem toda a área pastejada, levando à mai or compactação e uniformidade do estado físico do solo, contribuindo, assim, para a redução da variação e obtenção de diferenças significativas das variáveis analisadas. Segundo os resultados, após seis meses de instal ação do sistema de pastejo, ocorreu uma diminuição da variação, bem como tendência de homogeneização do estado de compactação da área. Similar tendência dos val ores de capacidade de suporte no decorrer de um experimento foi apresentada por Horn (2003).

Grande variação também foi verificada nos val ores de densidade do sol o em cada cicl o de pastejo (Figura 3). Vários estudos indicam que a $\sigma_{p}$ é 
influenciada pela densidade do solo (Silva et al., 2000; Canarache et al., 2000; Silva et al., 2002; I mhoff et al., 2004). A redução da variação dos dados de $D_{s}$ condicionou a obtenção de diferenças significativas noterceiro ciclo de pastejo. Foi possível perceber uma homogeneização nos valores deste indicador com o decorrer do tempo do experimento, corroborando os dados de Bell et al. (1997) e de Twerdoff et al. (1999) em sistemas pastoris.

O modelo não-linear, proposto por $\mathrm{McNabb} \&$ Boersma (1993), foi o que melhor ajustou os dados das curvas de compressão nos sistemas avaliados, conforme resultados da análise (Quadro 2).

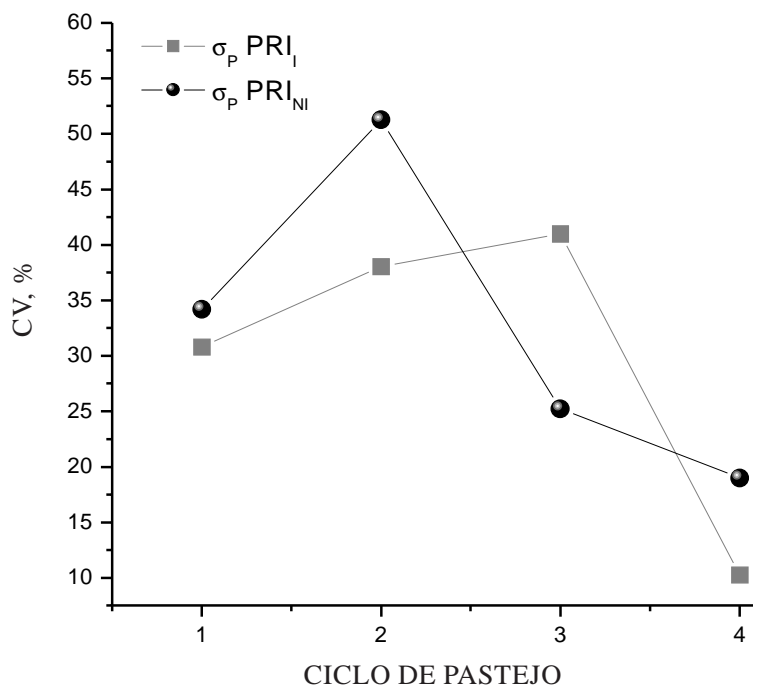

Figura 2. Coeficiente de variação da pressão de preconsolidação do solo $\left(\sigma_{\mathrm{p}}\right)$ em cada ciclo sob os sistemas de pastejo rotacionado intensivo irrigado $\left(P R I_{1}\right)$ e não irrigado $\left(P R I_{N I}\right)$.
O modelo explicou 88 e $94 \%$ da variação dos dados de densidade do solo nos sistemas de PRI, e $\mathrm{PRI}_{\mathrm{NI}}$, respectivamente. Todos os parâmetros foram significativos $(P<0,0001)$, visto que o intervalo de confiança do modelo (limite superior e inferior) não incluiu o valor zero (Quadro 2).

A partir do modelo, foram construídas as curvas de compressão uniaxial estimadas para os sistemas de $P R I_{1}$ e PRI ${ }_{\mathrm{NI}}$ (Figura 4).

As curvas decompressão uniaxial do sol orefletem as diferenças no processo de compactação dos solos

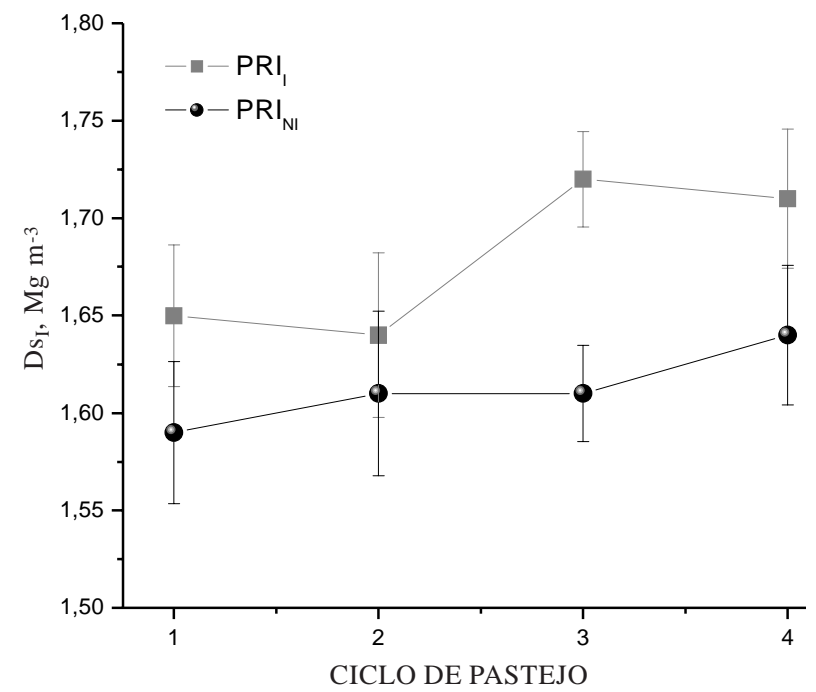

Figura 3. Valores médios de densidade do solo $\left(D_{s}\right)$ em cada ciclo sob os sistemas de pastejo rotacionado intensivo irrigado $\left(P R I_{1}\right)$ e não irrigado $\left(P R I_{N I}\right)$. Barras verticais indicam a diferença mínima significativa a $5 \%$ entre os sistemas de $P R I_{1}$ e $P R I_{N I}$.

Quadro 2. Resultados da regressão não-linear para o modelo: In $D_{s}=\ln \left(D_{s o *} \delta_{i}\right)-\left(a+b_{*} \sigma+C * \delta_{c}\right) *\left(1-e^{-d *} \sigma\right)$ sob os sistemas de pastejo rotacionado intensivo irrigado $\left(P R I_{I}\right)$ e não irrigado $\left(P R I_{N I}\right)$

\begin{tabular}{|c|c|c|c|c|}
\hline \multirow{2}{*}{ Parâmetro } & \multirow{2}{*}{ Valor estimado } & \multirow{2}{*}{ Erro-padrão } & \multicolumn{2}{|c|}{ Intervalo de confiança } \\
\hline & & & Limite inferior & Limite superior \\
\hline & \multicolumn{4}{|c|}{$P R I_{1}^{(1)}$} \\
\hline$D_{\text {so }}$ & 1,683 & 0,004 & 1,675 & 1,692 \\
\hline$a$ & $-0,072$ & 0,006 & $-0,0843$ & $-0,059$ \\
\hline $\mathrm{b}$ & $-0,045$ & 0,005 & $-0,056$ & $-0,034$ \\
\hline $\mathrm{C}$ & 5,547 & 0,961 & 3,658 & 7,436 \\
\hline \multirow[t]{2}{*}{$d$} & 0,229 & 0,021 & 0,189 & 0,271 \\
\hline & \multicolumn{4}{|c|}{$P R I_{N I}(2)$} \\
\hline $\mathrm{D}_{\mathrm{so}}$ & 1,618 & 0,003 & 1,612 & 1,624 \\
\hline a & $-0,076$ & 0,005 & $-0,086$ & $-0,066$ \\
\hline $\mathrm{b}$ & $-0,049$ & 0,004 & $-0,058$ & $-0,041$ \\
\hline c & 5,134 & 0,659 & 3,838 & 6,430 \\
\hline d & 0,204 & 0,014 & 0,176 & 0,232 \\
\hline
\end{tabular}

$\mathrm{D}_{\mathrm{s}}=$ densidade do solo $\left(\mathrm{Mg} \mathrm{m}^{-3}\right) ; \mathrm{D}_{\mathrm{so}}=$ densidade inicial estimada pelo modelo $\left(\mathrm{Mg} \mathrm{m}^{-3}\right) ; \delta_{\mathrm{i}}=$ densidade inicial/densidade média; $\delta_{\mathrm{c}}=$ $\left(\delta_{i}-1\right) \times\left(\right.$ densidade a $\left.0 \mathrm{MPa}, \mathrm{Mg} \mathrm{m}^{-3}\right) ; \sigma=$ pressão aplicada, $\mathrm{kPa} ; \mathrm{a} ., \mathrm{b}, \mathrm{c}, \mathrm{d}=$ parâmetros empíricos do modelo.

${ }^{(1)} \mathrm{F}=62581,1 ; \mathrm{P}<0,0001 ; \mathrm{R}^{2}=0,88(\mathrm{n}=440)$. (2) $\mathrm{F}=103147 ; \mathrm{P}<0,0001 ; \mathrm{R}^{2}=0,94(\mathrm{n}=440)$. 


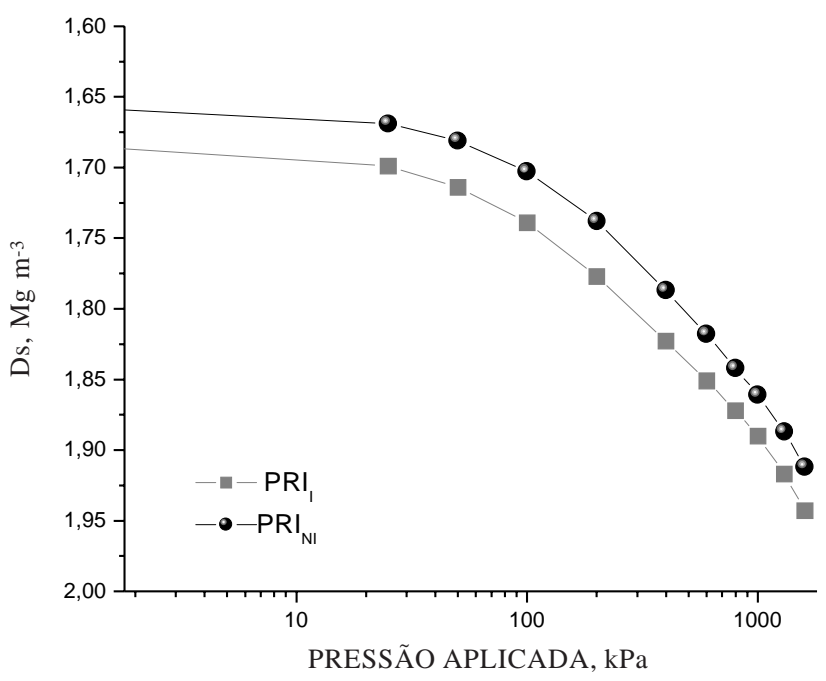

Figura 4. Curvas médias de compressão uniaxial estimadas sob os sistemas de pastejo rotacionado intensivo irrigado $\left(P R I_{1}\right)$ e não irrigado $\left(P R I_{N I}\right)$.

em cada área de pastejo. Esta diferença é observada no deslocamento da curva de compressão uniaxial para valores superiores de densidade do solo no sistema de PRI.

O deslocamento no sistema de $\mathrm{PRI}$, pode ter sido condicionado pela maior umidade do solo no momento do pisoteio do gado, ou seja, pela implementação da técnica da irrigação do sol o neste sistema. De acordo com Larson et al. (1980), à medida que a umidade aumenta, as curvas de compressão são geralmente desl ocadas para a região de maior densidade do solo. A umidade do solo é o fator que determina a magnitude de deformação que pode ocorrer no solo, quando determinado estresse é aplicado (McNabb \& Boersma, 1996; SánchesGirón et al., 1998; Mosaddeghi et al., 2000).

\section{CONCLUSÕES}

1. Os resultados comprovaram a hipótese de que houve diferença na compressibilidade do sol o sob os sistemas de pastejo rotacionado intensivo irrigado e não irrigado.

2. A maior compactação inicial verificada no sistema de pastejo rotacionado intensivo irrigado favoreceu o desl ocamento das curvas de compressão uniaxial para valores superiores de densidade do solo.

3. A pressão de preconsolidação foi significativamente superior no quarto ciclo de pastejo no sistema de pastejo rotacionado intensivo irrigado em relação ao sistema pastejo rotacionado intensivo não irrigado.

\section{LITE RATURA CITADA}

ABAYE, A.O.; ALLEN, V.G. \& FONTENOT, J .P. Grazing sheep and cattletogether or separately: Effect on soils and plants. Agron. J ., 89:380-386, 1997.

BLAKE, G.R. \& HARTGE, K.H. Bulk density. In: KLUTE, A., ed. Methods of soil analysis: Physical and mineralogical methods. 2.ed. Madison, American Society of Agronomy, 1986. p.363-375.

BELL, M.J .; BRIDGE, B.J .; HARCH, G.R. \& ORANGE, D.N. Physical rehabilitation of degraded Krasnozems using ley pastures. Aust. J . Soil Res., 35:1093-1113, 1997.

BETTERIDGE, K.; MACKAY, A.D.; SHEPHERD, T.G.; BARKER, D.J .; BUDDING, P.J .; DEVANTIER, B.P. \& COSTALL, D.A. Effect of cattle and sheep treading on surface configuration of a sedimentary hill soil. Aust. J . Soil Res., 37:743-760, 1999.

CANARACHE, A.; HORN, R. \& COLIBAS, I. Compressibility of soils in a long term field experiment with intensive deep ripping in Romania. Soil Till. Res., 56:185-196, 2000.

CASAGRANDE, A. The determination of the pre-consolidation load and its practical significance. In: Conference on soil mechanics and foundation engineering, Cambridge, 1936. Proceedings, Cambridge, ICSMFE, 1936. p.60-64.

CHANASYK, D.S. \& NAETH, M.A. Grazing impacts on bulk density and soil strength in the foothills fescue grasslands of Alberta, Canada. Can. J . Soil Sci., 75:551-557, 1995.

DEFOSSEZ, P. \& RICHARD, G. Models of soil compaction due totraffic and their evaluation. Soil Till. Res., 67:41-64, 2002.

DIAS J ÚNIOR, M.S. \& PIERCE , F.J . O processo de compactação do solo e sua modelagem. R. Bras. Ci. Solo, 20:175-182, 1996.

EMPRESA BRASILEIRA DE PESQUISA AGROPECUÁRIA EMBRAPA. Centro Nacional de Pesquisa de Solos. Sistema Brasileiro de Classificação de Solos, Brasília, Produção de Informação, Rio de J aneiro, Embrapa Solos, 1999. 412p.

FERRERO, A.F. Effect of compaction simulating cattletrampling on soil physical characteristics in woodland. Soil Till. Res., 19:319-329, 1991.

HORN, R. Stress-strain effects in structured unsaturated soils on coupled mechanical and hydraulic processes. Geoderma, 116:77-88, 2003.

HORN, R. \& LEBERT, M. Soil compactability and compressibility. In: SOANE, B.D. \& van OUWERKERK, C., eds. Soil compaction in crop production. Amsterdam, Elsevier, 1994. p.45-69.

IMHOFF, S.; SILVA, A.P. \& FALLOW, D. Susceptibility to compaction, load support capacity and soil compressibility of Hapludox. Soil Sci. Soc. Am. J ., 68:17-24, 2004.

J ORAJ URIA, D.; DRAGHI, L. \& ARAGON, A. The effect of vehicle weight on the distribution of compaction with depth and the yield of Loliun / Trifolium grassland. Soil Till. Res., 41:1-12, 1997. 
KLUTE, A. Water retention: laboratory methods. In: KLUTE, A., ed. Methods of soil analysis: Physical and mineralogical methods. 2.ed. Madison, American Society of Agronomy, 1986. p.635-660.

KONDO, M.K.\& DIAS J ÚNIOR, M.S. Compressibilidade de três Latossolos em função da umidade e uso. R. Bras. Ci. Solo, 23:211-218, 1999.

LARSON, W.E.; GUPTA, S.C. \& USECHE, R.A. Compression of agricultural soils from eight soil orders. Soil Sci. Soc. Am. J ., 44:450-457, 1980.

MACNABB, D.H. \& BOERSMA, L. Evaluation of the relationship between compressibility and shear strength of Andisols. Soil Sci. Soc. Am. J ., 57:923-929, 1993.

MACNABB, D.H. \& BOERSMA, L. Nonlinear model for compressibility of partly saturated soils. Soil Sci. Soc. Am. J ., 60:333-341, 1996.

MATHSOFT, Inc. Mathcad professional reference manual. Cambridge, Mathsoft Inc, 2000. 180p.

MAPFUMO, E.; CHANASYK, D.S.; NAETH, M.A. \& BARON, V.S. Soil compaction under grazing of annual and perennial forages. Can. J . Soil Sci., 79:191-199, 1999.

MOSADDEGHI, M.R.; HAJ ABBASI, M.A.; HEMMAT, A. \& AFYUNI, M. Soil compactibility as affected by soil moisture content and farmyard manure in central Iran. Soil Till. Res., 55:87-97, 2000.

NIE, Z.N.; WARD, G.N. \& MICHAEL, A.T. I mpact of pugging by dairy cows on pastures and indicators of pugging damage to pasture soil on south - Western Victoria. Aust. J . Agric. Soil Res., 52:37-43, 2001.

PROFFITT, A.P.B.; BENDOTTI, S.; HOWELL, M.R. \& EASTHAM, J. The effect of sheep trampling and grazing on soil physical properties and pasture growth for a Red Brown earth. Aust. J . Agric. Soil Res., 44:317-331, 1993.
SÁNCHES-GIRÓN, V.; ANDREU, E. \& HERNANZ, J.L. Response of five types of soil to simulated compaction in the form of confined uniaxial compression test. Soil Till. Res., 48:37-50, 1998.

SILVA, V.R.; REINERT, D.J . \& REICHERT, J.M. Suscepti bilidade à compactação de um Latossol o Vermel hoEscuro e de um Podzólico Vermelho-Amarelo. R. Bras. Ci. Solo, 24:239-249, 2000.

SILVA, V.R.; REINERT, D.J .; REICHERT, J .M. \& SOARES, J .M. Fatores controladores da compressibilidade de um Argissolo Vermel ho Amarelo distrófico arênico e de um Latossolo Vermel ho distrófico típico. I - Estado inicial de compactação. R. Bras. Ci. Solo, 26:1-8, 2002.

SILVA, A.P.; IMHOFF, S. \& CORSI, M. Evaluation of soil compaction in an irrigated short-duration grazing system. Soil Till. Res., 70:83-90, 2003.

SAS INSTITUTE - STATISTICAL Analysis System Institute. Procedure guide for personal computers. 5.ed. Cary, 1991.

STEPHENSON, G.R. \& VEIGEL, A. Recovery of compacted soil on pastures used for winter cattle feeding. J . Manag., 40:46-48, 1987

TANNER, C.B. \& MAMARIL, C.P. Pasture soil compaction by animal traffic. Agron. J ., 51:329-331, 1959.

TWERDOFF, D.A.; CHANASYK, D.S.; MAPFUMO, E.; NAETH, M.A. \& BARON, V.S. I mpacts of forage grazing and cultivation on near-surface relative compaction. Can. J. Soil Sci., 79:465-471, 1999.

WILLATT, S.T. \& PULLAR, D.M. Changes in soil physical properties under grazes pastures. Aust. J . Soil Res., 22:343348, 1983.

ZIMMER, A.; SILVA, M.P. \& MAURO, R. Sustentabilidade e impactos ambientais da produção animal em pastagens. In: PEIXOTO, A.M.; MOURA, J .C.; PEDREIRA, C.G.S. \& FARIA, V.P., eds. Inovações tecnológicas no manejo de pastagens. Piracicaba, FEALQ, 2002. p.31-58. 